\title{
Fabrication of Submicrocrystalline Structure in Solid Solutions by Radiation-Induced Phase Transition
}

\author{
V. A. Ivchenko \\ Ural Federal University, Yekaterinburg, 620002 Russia \\ Institute of Electrophysics, Ural Branch, Russian Academy of Sciences, Yekaterinburg, 620216 Russia \\ e-mail:ivchenko@iep.uran.ru \\ Received January 14, 2013
}

\begin{abstract}
The method of field ion microscopy was used to study the submicrocrystalline structure of the surface of PdCuAg solid solutions obtained after irradiation with $\mathrm{Ar}^{+}$ions. It was shown that there are radiationinduced structural phase transitions in the subsurface volume of solid solutions irradiated by gas ions with an energy of $20 \mathrm{keV}$ and a fluence of $10^{18} \mathrm{ion} / \mathrm{cm}^{2}$. The regimes were determined of radiation treatment of structural alloys Pd50Cu30Ag20 in which a microduplex-type structure with the phases' particle sizes being up to $100 \mathrm{~nm}$ with the necessary set of properties arises in the subsurface volume.
\end{abstract}

DOI: $10.1134 / \mathrm{S} 1063785013050052$

Modification of the surface of materials that occurs during irradiation by charged particles is done deliberately to achieve certain physical and mechanical properties of the surface. A significant change in the atomic structure of the surface may be caused by the phase structural changes that occur as a result of radiation exposure. By varying the parameters of the effect of radiation impact (energy and fluence of irradiation), we can obtain necessary changes in the atomic crystal structure of solid solutions in surface layers of a certain depth.

The aim of this work is to create a necessary submicrostructure in the subsurface volume of $\mathrm{PdCuAg}$ structural alloys using nonstandard thermomechanical treatments - in particular, implantation of gas ions upon a change in irradiation regimes. The objects of research are used as low-current sliding contacts in a special instrument.

In this respect, it is an important issue in the study of radiation material science during study of ion implanted structural materials to determine the structural and phase composition of thin surface layers. Therefore, the method of field ion microscopy (FIM) is used for precise study of the structural phase transitions occurring in subsurface volume of $\mathrm{PdCuAg}$ after irradiation by beams of charged particles $\left(\mathrm{Ar}^{+}\right)$. The capabilities of FIM allow us to investigate the real structure of the crystal lattice of solid solutions at the level of individual atoms, to work with an atomically clean surface at cryogenic temperatures, and at the same time to analyze the atomic structure of an object in a volume by controlled serial removal of surface atoms by an electric field. Measurement of the depth of the structural phase transition and the "thickness" of the modified surface volume of the material and the mechanism itself occurring under the effect of irradiation on the material is important to this work's goals.

Preliminarily deformed by $70 \%$ and annealed for $1 \mathrm{~h}$ at $T=1123 \mathrm{~K}$ (then cooled by water immersion) samples of Pd50Cu30Ag20 (wt \%) structural alloy were objects of interaction with a charged $\mathrm{Ar}^{+}$beam. (According to the phase diagram, the critical temperature of the phase transformation in the ternary solid solutions of $\mathrm{Pd} 50 \mathrm{Cu} 30 \mathrm{Ag} 20$ is $873 \mathrm{~K}$.)

Samples that were intended for investigation FIM were prepared in the form of needle-shaped emitters with a radius of curvature at the apex of $30-50 \mathrm{~nm}$ from pre-heat-treated alloy-wire billets by electrochemical polishing. Field emitters that have been certified for ion implantation have an atomically smooth surface, almost hemispherical and formed by in situ field evaporation of surface atoms.

$\mathrm{An} \mathrm{Ar}^{+}$ion beam is formed by the source basing of reflecting discharge with a hollow cold cathode. The maximum ion energy was $E=20 \mathrm{keV}$. Limits of the ion current density was $j=200-340 \mu \mathrm{A} / \mathrm{cm}^{2}$. This corresponded to a change of the sample temperature during irradiation in the range of $T=450-700 \mathrm{~K}$. To control the dose, a precertified object of study was placed in a field ion microscope in a water-cooled Faraday cup. Measuring the ion current density and fluence was performed by an automated system.

Then implanted samples were again placed in the chamber of FIM. The structural state of the alloy in the bulk was analyzed by recording of ion images of surface during controlled removal of atomic layers. The total number of irradiated specimens investigated in FIM was about 100 pieces. A field ion microscope was equipped with a microchannel intensifier. Solid 
nitrogen $(T=63 \mathrm{~K})$ was usually the refrigerant. Spectrally pure neon was used as the imaging gas.

Analysis of the neon image of an atomically clean surface of Pd50Cu30Ag20 (wt \%) ternary solid solutions showed that the alloy approved for subsequent implantation has the structure of a disordered FCC solid solution.

There is a neon image of an atomically clean surface of Pd50Cu30Ag20 (wt \%) ternary solid solution certified for subsequent implantation of argon ions in Fig. 1a. This is a typical surface micromap of the microdisordered FCC solid solution [1]. The crystal is orientated along the $\langle 111\rangle$ direction along the axis of the sample almost perpendicular to the micrograph. In addition to the central facet (111), there are $\{100\}$, $\{110\}$, and $\{113\}$ planes in the micrograph. This is characteristic in the style of facets for ion images of crystals with an FCC lattice.

A structure state of triple solid solution has changed after irradiation by argon ions with an energy of $20 \mathrm{keV}$, current density of $\sim 340 \mu \mathrm{A} / \mathrm{cm}^{2}$, and a fluence of $10^{18}$ ion $/ \mathrm{cm}^{2}$, as it is clearly shown in Fig. $1 \mathrm{~b}$ (an atomically clean surface of the irradiated alloy is shown in Fig. 1b). There is a superstructure pole (001) of $\mathrm{PdCu}$ ordered phase particles in the micrograph. Therefore, it is safe to state that there is a structural phase transition in the subsurface volume of Pd50Cu30Ag20 ternary solid solutions initiated by argon ion implantation (which was previously shown in [1]).

The mechanism of phase transformations in the $\mathrm{PdCuAg}$ system after thermomechanical treatment is studied in [2-4]. It has been shown that, in this system, in the early stages of the structural transformation occurs intermittently decomposition with the formation of the ordered phase $\mathrm{PdCu}$ (type B2 superstructure) and the solid solution phase AgPd (FCC lattice). At the moment of the appearance of the phase $\mathrm{PdCu}$ is ordered according to type B2 with a high degree of long-range order in the grains. Microcrystals that form during intermittent dissolution of triple supersaturated solid solution take the form of plates. Cellular formation of the $\mathrm{PdCu}$ ordered phase and $\mathrm{AgPd}$ phase was established. Moreover, the resulting cell contains one particle of each phase. It is shown in [3] that the high strength and plastic properties of the $\mathrm{PdCuAg}$ alloys were obtained by forming in the material so-called microduplex structure. Analysis of the spatial distribution of submicrocrystalline phases (the size of individual grains cannot exceed $100 \mathrm{~nm}$ ) in the amount of material showed that the grains of the BCC phase formed between the boundaries of high coherence, as in some special cases. The forms, structure, and volume fraction of the phases that make up the microduplex structure were established.

Therefore, the goal was to obtain exposure modes for the modified subsurface volume microduplex type structure. In [5], it was found that, depending on the size of the ion current density under other constant irradiation parameters, the size of the surface where
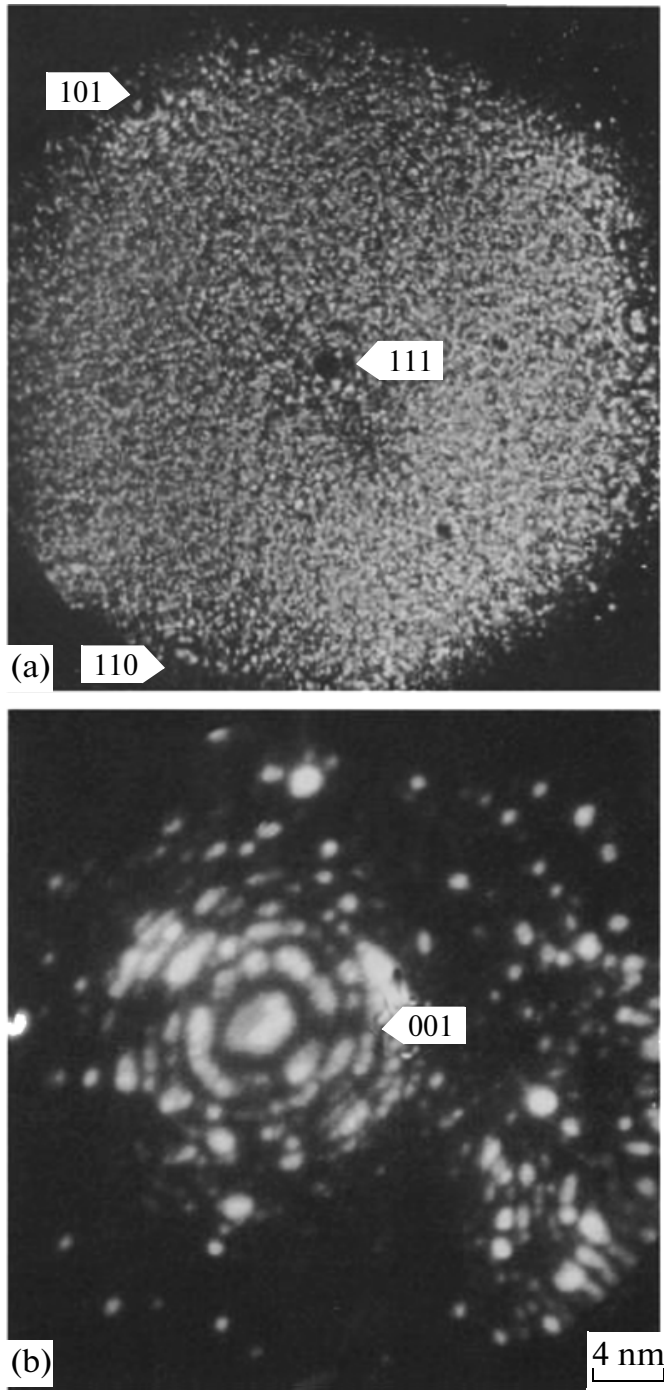

Fig. 1. (a) Neon image of an atomically clean surface of Pd50Cu30Ag20 (wt \%) ternary solid solutions certified for subsequent irradiation with argon ions; (b) ionic contrast particles ordered according to phase $\mathrm{PdCu}$ resulting from exposure (as indicated by the arrow (001)).

there is a structural phase transformation also changed. At a current density in the beam of $300 \mu \mathrm{A} / \mathrm{cm}^{2}$, the phase transformation extended to a depth of $\sim 100 \mathrm{~nm}$ from the irradiated surface; at $j=$ $340 \mu \mathrm{A} / \mathrm{cm}^{2}$, the phase transformation extended to a depth of at least $600 \mathrm{~nm}$ (Fig. 2). It should be noted that for $j=200 \mu \mathrm{A} / \mathrm{cm}^{2}$ no intermittent collapse in triple solid solution occurs.

In the process of field evaporation of atoms from the surface of the ion-implanted $\mathrm{PdCuAg}(j=$ $340 \mu \mathrm{A} / \mathrm{cm}^{2}$ ) in situ, vacancy clusters (micropores) were observed in the surface volume and their size and shape were determined. These microscopic pores were observed immediately after the removal of the first atomic layers of the irradiated surface and to a depth of $\sim 80 \mathrm{~nm}$ in volume at a beam current density of 

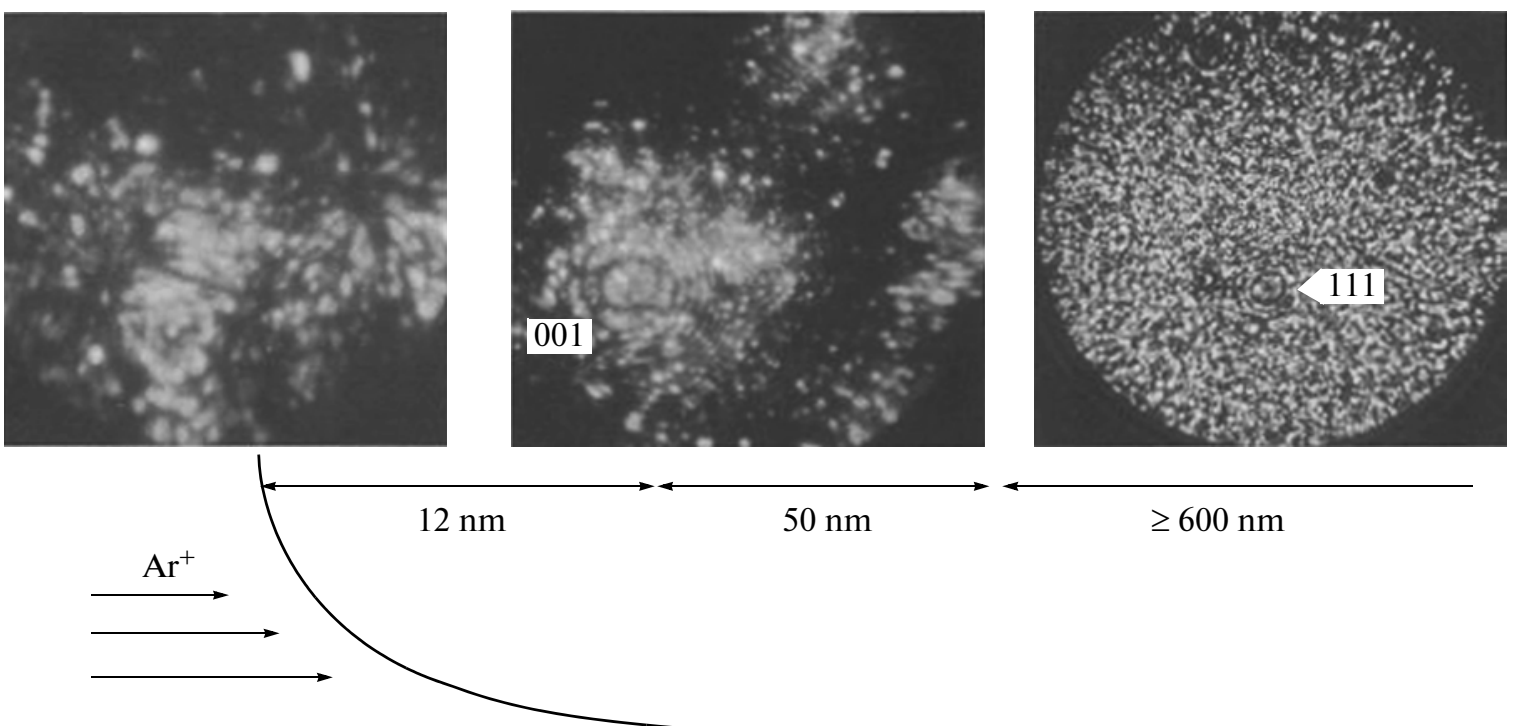

Fig. 2. Scheme of Pd50Cu30Ag20 (wt \%) ternary solid solution surface structural state condition measurement after irradiation with $\left(j=340 \mu \mathrm{A} / \mathrm{cm}^{2}, F=10^{18} \mathrm{ion} / \mathrm{cm}^{2}\right)$ during moving atomic layers from the irradiated surface for corresponding distance.

$340 \mu \mathrm{A} / \mathrm{cm}^{2}$, which was determined by calculating the evaporating electric field of the surface atomic layers. It is known from [6] that argon ions at the beam energies employed have a projected range of not more than $15 \mathrm{~nm}$ in pure components of the studied solid solution.

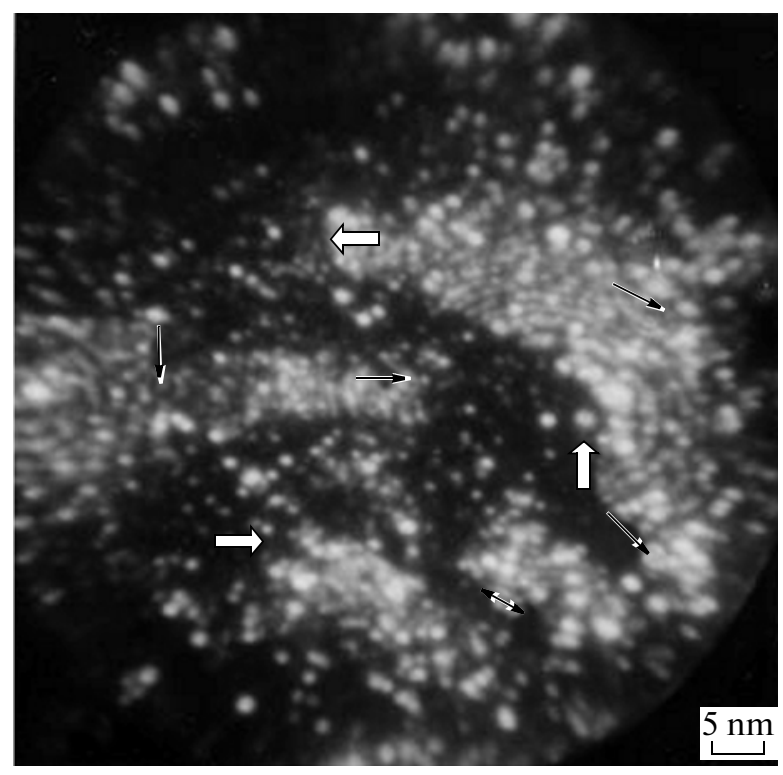

Fig. 3. Ionic contrast of microduplex type structure obtained after irradiation of surface Pd50Cu30Ag20 (wt \%) by $\mathrm{Ar}^{+}$with $F=10^{18}$ ion $/ \mathrm{cm}^{2}$, with $j=300 \mu \mathrm{A} / \mathrm{cm}^{2}$; black arrows indicate $\mathrm{PdCu}$ phase particles ordered by type B2; white arrows indicate $\mathrm{AgPd}$ (solid solution) phase particles.
This shows that pore swelling in the material did not occur due to the presence of implanted ions of argon, which could become centers of origin of micropores, but rather due to metastability of the ternary structure of the solid solution to intermittent degradation during irradiation. Detailed analysis of the experimental data suggests that, as a result of ion implantation, there is a continuous increase in the migration of interstitials and vacancies to sinks and association of single vacancies in vacancy clusters (micropores). Obviously, the process of vacancy clusters accelerates a structural phase transition in the triple solid solution-namely, intermittent collapse. Such radiation-induced phase transformation naturally leads to a significant change of the surface properties of the PdCuAg alloy. It is also obvious that these parameters of ion implantation prohibit obtaining a microduplex type of structure in the modified surface material volume. Moreover, the occurrence of micropores affects the mechanical properties of the surface.

In changing regimes of ion implantation (in a range only to the value of the ion current), it was found that, for $j=300 \mu \mathrm{A} / \mathrm{cm}^{2}$, a microduplex type structure arose in triple solid solution $\mathrm{PdCuAg}$, but the amount of the phase precipitates was comparable and even slightly less than the amount of the phases after thermomechanical effects (Fig. 3).

In an FIM study of ion-implanted $\mathrm{PdCuAg}$, a phase-structural transition was found to the subsurface volume of the material and the regime was determined of radiation treatment of structural alloys such as $\mathrm{Pd} 50 \mathrm{Cu} 30 \mathrm{Ag} 20$, in which, in the a surface microduplex type structure appears (with a particle size of phases up to $100 \mathrm{~nm}$ ) in the subsurface volume with 
a desired combination of properties of the investigated structural materials. The size of the surface of the subsurface volume was determined in which there was a structural-phase transition depending on the density of the beam current.

The dependence of the modification of the structure of the surface of the implanted alloy (with evaluation of the depth of the modified surface, which has undergone structural phase transformation) of irradiation parameters (irradiation energy, ion current density, fluence, temperature) has been determined.

Acknowledgments. The work was supported by the Ural Branch of the Russian Academy of Sciences, Program no. 5 "Physics of New Materials and Structures."

\section{REFERENCES}

1. V. A. Ivchenko and N. N. Syutin, Appl. Surf. Sci. 87/88, 257 (1995).

2. N. N. Syutkin, V. A. Ivchenko, and S. I. Noritsyn, Fiz. Met. Metalloved. 57 (1984).

3. N. N. Syutkin, V. A. Ivchenko, A. B. Telegin, and S. I. Noritsyn, Fiz. Met. Metalloved. 60, 607 (1985).

4. N. N. Syutkin, V. A. Ivchenko, and A. B. Telegin, Fiz. Met. Metalloved. 62, 965 (1986).

5. V. A. Ivchenko, V. V. Ovchinnikov, B. Yu. Gooborodsky, and N. N. Syutkin, Surf. Sci. 384, 46 (1997).

6. A. F. Burenkov, F. F. Komarov, M. A. Kumakhov, and M. M. Temkin, Spatial Distribution of Energy Liberated in Atomic Collision Cascades in Solids (Energoatomizdat, Moscow, 1985) [in Russian].

Translated by A.V. Viazovtcev 